\title{
Chromatic Signatures in the Microlensing of Gamma-Ray Burst Afterglows
}

\section{Citation}

Granot, Jonathan, and Abraham Loeb. 2001. "Chromatic Signatures in the Microlensing of Gamma-Ray Burst Afterglows." The Astrophysical Journal 551 (1): L63-66. https:// doi.org/10.1086/319843.

\section{Permanent link}

http://nrs.harvard.edu/urn-3:HUL.InstRepos:41393281

\section{Terms of Use}

This article was downloaded from Harvard University's DASH repository, and is made available under the terms and conditions applicable to Other Posted Material, as set forth at http:// nrs.harvard.edu/urn-3:HUL.InstRepos:dash.current.terms-of-use\#LAA

\section{Share Your Story}

The Harvard community has made this article openly available.

Please share how this access benefits you. Submit a story.

Accessibility 


\title{
Chromatic Signatures in the Microlensing of GRB Afterglows
}

\author{
Jonathan Granot ${ }^{1}$ and Abraham Loeb ${ }^{2}$
}

\begin{abstract}
We calculate the radial surface brightness profile of the image of a GammaRay-Burst (GRB) afterglow. The afterglow spectrum consists of several power-law segments separated by breaks. The image profile changes considerably across each of the spectral breaks. It also depends on the density profile of the ambient medium into which the GRB fireball propagates. Gravitational microlensing by an intervening star can resolve the afterglow image. We calculate the predicted magnification history of GRB afterglows as a function of observed frequency and ambient medium properties. We find that intensive monitoring of a microlensed afterglow lightcurve can be used to reconstruct the parameters of the fireball and its environment, and provide constraints on particle acceleration and magnetic field amplification in relativistic blast waves.
\end{abstract}

Subject headings: gamma rays: bursts-gravitational lensing

\section{Introduction}

The fireball of a Gamma-Ray Burst (GRB) afterglow is predicted to appear on the sky as a ring (in the optical band) or a disk (at low radio frequencies) that expands laterally at a superluminal speed, $\sim \Gamma c$, where $\Gamma \gg 1$ is the Lorentz factor of the relativistic blast wave which emits the afterglow radiation (Waxman 1997; Sari 1998; Panaitescu \& Mészáros 1998; Granot, Piran, \& Sari 1999a,b). Days after the GRB trigger, the physical radius of the afterglow image $(\sim$ the fireball radius over $\Gamma$ ) translates to an angular size of order a micro-arcsecond ( $\mu$ as) at a cosmological distance. Coincidentally, this image size is comparable to the Einstein angle of a solar mass lens at a cosmological distance,

$$
\theta_{\mathrm{E}}=\left(\frac{4 G M_{\mathrm{lens}}}{c^{2} D}\right)^{1 / 2}=1.6\left(\frac{M_{\mathrm{lens}}}{1 M_{\odot}}\right)^{1 / 2}\left(\frac{D}{10^{28} \mathrm{~cm}}\right)^{-1 / 2} \mu \mathrm{as},
$$

where $M_{\text {lens }}$ is the lens mass, $D \equiv D_{\mathrm{os}} D_{\mathrm{ol}} / D_{\mathrm{ls}}$ is the ratio of the angular-diameter distances between the observer and the source, the observer and the lens, and the lens and the source

\footnotetext{
${ }^{1}$ Racah Institute, Hebrew University, Jerusalem 91904, Israel; jgranot@merger.fiz.huji.ac.il

${ }^{2}$ Harvard-Smithsonian Center for Astrophysics, 60 Garden Street, Cambridge, MA 02138; aloeb@cfa.harvard.edu
} 
(Schneider, Ehlers, \& Falco 1992). Loeb \& Perna (1998) argued that microlensing by stars could therefore be used to resolve the photospheres of GRB afterglows.

Recently, Garnavich, Loeb, \& Stanek (2000) have reported the possible detection of a microlensing magnification feature in the optical-infrared light curve of GRB 000301C. The achromatic transient feature is well fitted by a microlensing event of a $0.5 M_{\odot}$ lens separated by an Einstein angle from the source center, and resembles the prediction of Loeb \& Perna (1998) for a ring-like source image with a narrow fractional width $(\sim 10 \%)$. Alternative interpretations relate the transient achromatic brightening to a higher density clump into which the fireball propagates (Berger et al. 2000), or to a refreshment of the decelerating shock either by a shell which catches up with it from behind or by continuous energy injection from the source (e.g., Zhang \& Mészáros 2000). In order to regard these alternatives as less plausible, it is essential to identify the unique temporal and spectral characteristics of a microlensing event.

Gravitational lensing of a point source is achromatic due to the equivalence principle. However, differential magnification by an achromatic lens could still produce a chromatic magnification signal if the source is extended and its image looks different at different photon frequencies (see, e.g. Loeb \& Sasselov 1995). Should a microlensing event of GRB afterglows be achromatic? Since the image profile of an afterglow does depend on frequency (Sari 1998; Panaitescu \& Mészáros 1998; Granot, Piran, \& Sari 1999a,b), its magnification lightcurves must exhibit a predictable level of chromaticity. In $\S 2$ we will demonstrate that the surface brightness profile of an afterglow image changes considerably with frequency across all spectral breaks in its broken power-law spectrum. We will therefore show in $\S 3$ that the magnification history of an intervening microlens would depend on frequency across spectral breaks. However, within each power-law segment of the afterglow spectrum, the magnification would remain achromatic, in rough consistency with the sparse optical-infrared observations of GRB 000301C. This spectral behavior offers a fingerprint that can be used to identify a microlensing event and distinguish it from alternative interpretations. It can also be used to constrain the relativistic dynamics of the fireball and the properties of its gaseous environment, as well as the length scale required for particle acceleration and magnetic field amplification in relativistic shocks.

\section{Frequency Dependence of Afterglow Images}

The surface brightness profile (SBP) of an afterglow depends on the underlying hydrodynamics of the fireball, as well as on the energy distribution of shock-accelerated electrons. In this Letter we adopt the model used by Granot \& Sari (2001, hereafter GS01) to describe afterglows. Here we provide a brief outline of this model, and refer the reader to Granot et al. (1999a,b) and GS01 for more details.

The hydrodynamics is described by the Blandford-McKee (1976) self-similar spherical solution, with a power-law external density profile, $\rho \propto R^{-k}$, for either $k=0$ or $k=2$, corresponding to a 
uniform interstellar medium (ISM) or a stellar wind environment, respectively. The number per unit energy of accelerated electrons is assumed to be a power law, $d N_{e} / d \gamma \propto \gamma^{-p}$ (for $\gamma>\gamma_{m}$ ), just behind the shock. Thereafter, the initial distribution evolves due to radiative and adiabatic losses. The emission mechanism is assumed to be synchrotron radiation, and the emissivity is integrated over the entire volume behind the shock front.

The afterglow image is limited to a circle on the sky, whose size grows as $t^{(5-k) / 2(4-k)}$, where $t$ is the observed time. The assumption of a spherical flow may also serve as an adequate description of a jetted flow, at sufficiently early times before the jet break time, $t_{j e t}$, when the Lorentz factor of the flow drops below the inverse of the jet opening angle (Rhoads 1997). In the case of a jet, the image at $t \gtrsim t_{j e t}$ is expected to be different than in the spherical case, and will no longer be circular for observers who are situated away from the jet axis. Deviations from sphericity might also result from hydrodynamic or plasma instabilities. For simplicity we focus here on a spherical fireball; the more complex cases mentioned above are left for a future study.

The spectrum of GRB afterglows consists of several power-law segments (PLSs) where the flux density $F_{\nu} \propto \nu^{\beta}$, which join at certain break frequencies (Sari, Piran \& Narayan 1998, hereafter SPN98; Granot, Piran \& Sari 2000, hereafter GPS00; GS01). The cooling time of a typical electron, $t_{c o o l}$, is initially smaller than the dynamical time, $t_{d y n}$, resulting in "fast cooling" (SPN98), while at latter times $t_{\text {cool }}$ becomes larger than $t_{d y n}$ and there is a transition to "slow cooling". The fast cooling stage is typically expected to last for the first hour (day) for an ISM (stellar wind) environment (SPN98; Chevalier \& Li 2000). Since the angular size of the source is very small at such early times, the source will remain $\ll \theta_{\mathrm{E}}$ and hence unresolved during the fast cooling regime of a typical microlensing event (or else be resolved only in a negligible fraction of all events for which the lens nearly coincides with the source center). We will therefore restrict our attention to the slow cooling regime. The slow cooling spectrum may assume one of three different shapes, depending on the ordering of the self-absorption frequency, $\nu_{a}$, with respect to $\nu_{m}<\nu_{c}$, where $\nu_{m}$ is the typical synchrotron frequency and $\nu_{c}$ is the cooling frequency (GS01). The most common ordering of the break frequencies is the one shown in Figure 1 with $\nu_{a}<\nu_{m}<\nu_{c}$. For clarity, we show a broken power law spectrum, even though in the actual spectrum obtained from our model the PLSs join smoothly, and the break frequencies are defined where the asymptotic power laws meet (e.g. Granot et al. 1999b). Altogether, there are five different PLSs that appear in the different slow cooling spectra, which correspond to $\beta=5 / 2,2,1 / 3,(1-p) / 2,-p / 2$. Due to the self-similar hydrodynamics, the surface brightness, normalized by its average value over the image, is independent of time within a given PLS $\beta$. For $\beta=(1-p) / 2$ and $\beta=-p / 2$, the value of $\beta$ depends on $p$. The values of $\beta$ in these two PLSs may therefore accidentally coincide with those of other PLSs. However, the SBP will still be different, since the underlying physics is different.

\footnotetext{
${ }^{3}$ This statement remains valid even when taking into account the fast cooling spectra, which include two additional PLSs corresponding to $\beta=11 / 8,-1 / 2$ (e.g. SPN98; GPS00), with only one exception: $\beta=1 / 3$, where the SBP is different between slow cooling and fast cooling, since the underlying physics is different.
} 
Although for these two PLSs the SBP depends on the value of $p$, we find that at any given time, the differences in the microlens magnification induced by the differences in the SBP, never exceed

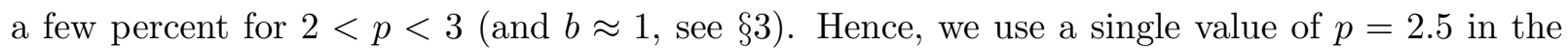
following.

Figure 2 shows the surface brightness, normalized by its average value, as a function of the normalized radius inside the image, $r$, for $k=0,2$ and $\beta=5 / 2,2,1 / 3,(1-p) / 2,-p / 2$. The SBPs for $k=0$ and $\beta=2,1 / 3,(1-p) / 2$ are taken from Granot et al. (1999a,b), while for the other values of $k$ and $\beta$, the SBPs are calculated in a similar manner, using the formalism of GS01. For both values of $k$, the image tends to be more uniform as $\beta$ increases and more ring-like (i.e. $\operatorname{dim}$ near the center and bright near the outer edge) at low values of $\beta$. For a given PLS (i.e. a given $\beta$ in Fig. 2), the image tends to be more uniform for a stellar wind environment $(k=2)$ as compared to an ISM environment $(k=0)$. For $\beta=-p / 2$ the surface brightness diverges at the outer edge of the image $(r=1)$, since the emission in this case is both optically thin and originates from a very thin layer behind the shock, leading to a relativistic version of the limb brightening effect (e.g. Sari 1998). f However, the physical size of this thin layer cannot be smaller than that of the transition layer just behind the shock front, where the electrons are accelerated and the magnetic field is amplified. As will be shown in $\$ 3$, the divergence of the SBP at $r=1$ leads to a very sharp spike in the magnification history. Introducing a finite width to the transition layer would eliminate the divergence of the SBP. However, a thin transition layer would still result in a sharp peak of the SBP near $r=1$, and a sharp peak in the magnification history, whereas a thick transition layer will result in a lower and smoother peak in both. Therefore, the sharpness of the magnification spike may set constraints on the physical processes of particle acceleration and magnetic field amplification in relativistic blast waves.

\section{Microlensing Histories}

Next we calculate the magnification histories of GRB afterglows due to microlensing by an intervening star at a cosmological distance. Since the effects of microlensing depend on the angular structure of the image in units of the Einstein angle $\theta_{\mathrm{E}}$ [Eq. (11)], we normalize all angular scales by $\theta_{\mathrm{E}}$. The angular radius of the afterglow image, in units of $\theta_{\mathrm{E}}$, is given by

$$
R_{s}(t)=R_{0} t_{\text {days }}^{(5-k) / 2(4-k)}
$$

where $t_{\text {days }}$ is the observed time in days and $R_{0}$ is the angular size of the image in units of $\theta_{\mathrm{E}}$ after one day. The angular separation between the center of the image and the lens, in units of $\theta_{\mathrm{E}}$, is

\footnotetext{
${ }^{4}$ The divergence scales as $(1-r)^{-1 / 2}$, since the intensity from an optically-thin shell is proportional to its geometric length along the direction to the observer. This limb brightening effect does not occur for the other optically-thin PLSs we consider, where the emission originates from the entire three-dimensional volume behind the shock. Only the fast-cooling electrons produce the radiation from a very thin layer just behind the shock front. Note that the diverging cusp makes a vanishing contribution to the average surface brightness of the afterglow image.
} 
denoted by $b$. The magnification of a uniform ring of fractional width $W$ (occupying the interval $1-W<r<1$ ) is given by (Loeb \& Perna 1998),

$$
\mu\left(R_{s}, W, b\right)=\frac{\Psi\left(R_{s}, b\right)-(1-W)^{2} \Psi\left[(1-W) R_{s}, b\right]}{1-(1-W)^{2}}
$$

where $\Psi\left(R_{s}, b\right)$ is the magnification of a uniform disk of radius $R_{s}$ (Schneider et al. 1992),

$\Psi\left(R_{s}, b\right)=\frac{2}{\pi R_{s}^{2}}\left[\int_{\left|b-R_{s}\right|}^{b+R_{s}} d R \frac{R^{2}+2}{\sqrt{R^{2}+4}} \arccos \frac{b^{2}+R^{2}-R_{s}^{2}}{2 R b}+H\left(R_{s}-b\right) \frac{\pi}{2}\left(R_{s}-b\right) \sqrt{\left(R_{s}-b\right)^{2}+4}\right]$,

and $H(x)$ is the Heaviside step function. In order to obtain the magnification of a radially symmetric image, one can either divide it into a large number of thin rings, or equivalently use the resulting integral formula for the frequency-dependent magnification (Witt \& Mao 1994),

$$
\mu_{\nu}\left(R_{s}, b\right)=\int_{0}^{1} 2 r d r\left[\Psi\left(r R_{s}, b\right)+\frac{r}{2} \frac{\partial \Psi}{\partial r}\left(r R_{s}, b\right)\right] \tilde{I}_{\nu}(r)
$$

where $\tilde{I}_{\nu} \equiv I_{\nu} /\left\langle I_{\nu}\right\rangle$ is the specific intensity normalized by its average value, $\left\langle I_{\nu}\right\rangle=\int_{0}^{1} 2 r d r I_{\nu}(r)$, as shown in Figure 2. Note that for a uniform disk $\left(\tilde{I}_{\nu}(r) \equiv 1\right)$, equation (5) gives $\mu_{\nu}=\Psi\left(R_{s}, b\right)$, as it should.

The magnification history for $b=1, k=0,2$ and $\beta=5 / 2,2,1 / 3,(1-p) / 2,-p / 2$, is shown in Figure 3. The peak in the magnification history is generally higher and sharper, and occurs at a slightly earlier time for a smaller spectral slope, $\beta$. This follows from the general trend of the SBP, which tends to be more sharply peaked near the outer edge of the image for lower values of $\beta$. At high values of $\beta$, especially in the optically-thick regime $(\beta=5 / 2,2)$, the magnification peak is relatively shallow and broad. For an ISM environment $(k=0)$, the peak magnification is somewhat higher and the differences in the magnification histories for different $\beta$ values are more pronounced than for a stellar wind environment $(k=2)$. These differences may allow to uncover the density profile of the circumburst medium from intense monitoring of the microlensing 'bump' in an afterglow light curve. Unfortunately, the quality of the observational data for GRB 000301C is not sufficient for this purpose.

We now consider the effects of microlensing on the global spectrum. For clarity, this is illustrated in Figure 1, on the spectrum therein. The spectrum consists of several PLSs: $\beta_{1}=2$, $\beta_{2}=1 / 3, \beta_{3}=(1-p) / 2$ and $\beta_{4}=-p / 2$, with different magnification histories, $\mu_{i}(t)(i=1, \ldots, 4)$. This causes each PLS to shift by a different time-dependent factor, as illustrated by the thin solid line in Figure 1 for $t_{\text {days }}=1$. The different magnification in each PLS generates a shift in the location of the break frequencies compared to their unlensed values, as illustrated in the insert at the top right corner of Figure 1. The value of the $i$-th break frequency (numbered from low to high frequencies, as are the PLSs) will shift by a factor of $\left[\mu_{i+1}(t) / \mu_{i}(t)\right]^{1 /\left(\beta_{i}-\beta_{i+1}\right)}$. The largest shift occurs at $\nu_{c}$ since the change in the spectral slope across the break is rather small $\left(\beta_{3}-\beta_{4}=1 / 2\right)$ and the ratio $\mu_{4}(t) / \mu_{3}(t)$ reaches relatively high values (see the upper panel of Figure [3). 


\section{Discussion}

We have shown that the frequency dependence of the afterglow image profile (Fig. 2) introduces a specific level of chromaticity to the magnification history of GRB afterglows (Fig. 3). This unique fingerprint can be used to distinguish between microlensing and alternative hydrodynamic interpretations for the transient brightening in an afterglow light curve.

For example, if the GRB blast wave runs into a clump of gas along the line-of-sight (Berger et al. 2000), then brightening will occur first in the radio and later in the optical, in opposite order to the microlensing case. The chromaticity in the clump case is sensitive to the exact geometry of the clump and the sharpness of its boundary (which could send a reverse shock into the fireball). In another example, the brightening event may result from refreshment of the decelerating shock (Zhang \& Mészáros 2000). In this case, the break frequencies and peak flux of the spectrum in Figure 1, namely $\nu_{a}, \nu_{m}, \nu_{c}$ and $F_{\nu_{m}}$, will change by a factor of $f^{1 / 5}, f^{1 / 2}, f^{-1 / 2}$ and $f$ (or by $f^{-2 / 5}, f^{1 / 2}, f^{1 / 2}$ and $f^{1 / 2}$ ), respectively, for an ISM with $k=0$ (or stellar wind with $k=2$ ) environment, where $f$ is the ratio between the total energies in the forward shock after and before the refreshment. This behavior clearly differs from the microlensing prediction (see insert in Fig. 1). By itself, the magnification history of microlensing is already unique in that it is initially characterized by a constant positive offset as long as $R_{s}(t) \ll 1$ (Loeb \& Perna 1998). Such an initial positive offset does not appear naturally in either of these alternative interpretations.

Microlensing events are rare but precious. Only one out of roughly a hundred afterglows is expected to be strongly microlensed (Press \& Gunn 1973; Blaes \& Webster 1992; Koopmans \& Wambsganss 2000), although all afterglows are expected to be magnified at some weak level (Mao \& Loeb 2000). Detailed monitoring of a few strong microlensing events among the hundreds of afterglows detected per year by the forthcoming Swift satellite?, could be used to constrain the environment and the dynamics of relativistic GRB fireballs, as well as their magnetic structure and particle acceleration process (Fig. 3). Unfortunately, the data on the existing microlensing candidate GRB 000301C is not of sufficient quality to provide firm constraints on these parameters, although the $R$-band data seems to suggest $\beta=-p / 2$ and gives a somewhat better fit for $k=0$ (a uniform density environment) than for other parameter choices. This can be seen by crudely approximating the SBP as a ring. We find that for $k=0$, a fraction of $2 / 3(3 / 4)$ of the emission comes from a ring of fractional width $W=0.156(0.190)$ for $\beta=(1-p) / 2$ and $W=0.075(0.104)$ for $\beta=-p / 2$; while for $k=2, W=0.318$ (0.370) for $\beta=(1-p) / 2$ and $W=0.128$ (0.176) for $\beta=-p / 2$. The $k=0, \beta=-p / 2$ values get closest to the empirical best-fit values of $W=0.07$ using the R-band data (Garnavich et al. 2000). The statistical accuracy by which the unknown brightness profile of a future microlensed afterglow can be reconstructed through an intensive monitoring campaign, was analyzed in detail recently by Gaudi \& Loeb (2001).

\footnotetext{
${ }^{5}$ Planned for launch in 2003; see http://swift.sonoma.edu/
} 
This work was supported in part by the Horowitz foundation and the US-Israel BSF grant BSF-9800225 (for JG), and by NASA grants NAG 5-7039, NAG 5-7768, NSF grants AST-9900877, AST-0071019 and US-Israel BSF grant BSF-9800343 (for AL).

\section{REFERENCES}

Berger, E., et al. 2000, ApJ, 545, 56

Blaes, O. M., \& Webster, R. L. 1992, ApJ, 391, L63

Blandford, R. D. \& McKee, C. F. 1976, Phys. of fluids, 19, 1130

Chevalier, R.A., \& Li, Z.Y., 2000, ApJ, 536, 195

Garnavich, P.M., Loeb, A. \& Stanek, K.Z. 2000, ApJ, 544, L11

Gaudi, S., \& Loeb, A. 2001, ApJ, submitted; astro-ph/0102003

Granot, J., Piran, T. \& Sari, R. 1999a, ApJ, 513, 679

. 1999b, ApJ, 527, 236

. 2000, ApJ, 534, L163 (GPS00)

Granot, J., \& Sari, R. 2001, in preparation (GS01)

Koopmans, L. V. E., \& Wambsganss, J. 2000, MNRAS, in press; astro-ph/0011029

Loeb, A. \& Perna, R. 1998, ApJ, 495, 597

Loeb, A., \& Sasselov, D. 1995, ApJ, 449, L33

Mao, S. \& Loeb, A. 2000, ApJL in press; astro-ph/0010029

Panaitescu, A., \& Mészáros, P. 1998, ApJ, 493, L31

Press, W. H., \& Gunn, J. E. 1973, ApJ, 185, 397

Rhoads, J. E. 1997, ApJ, 487, L1

Sari, R. 1998, ApJ, 494, L49

Sari, R., Piran, T. \& Narayan, R. 1998, ApJ, 497, L17 (SPN98)

Schneider, P., Ehlers, J., \& Falco, E.E. 1992, Gravitational Lenses (Berlin; Springer), pp. 27, 313

Waxman, E. 1997, ApJ, 491, L19

Witt, H.J. \& Mao, S. 1994, ApJ, 430, 505 
Zhang, B., \& Mészáros, P. 2000, ApJL, submitted; astro-ph/0011133 


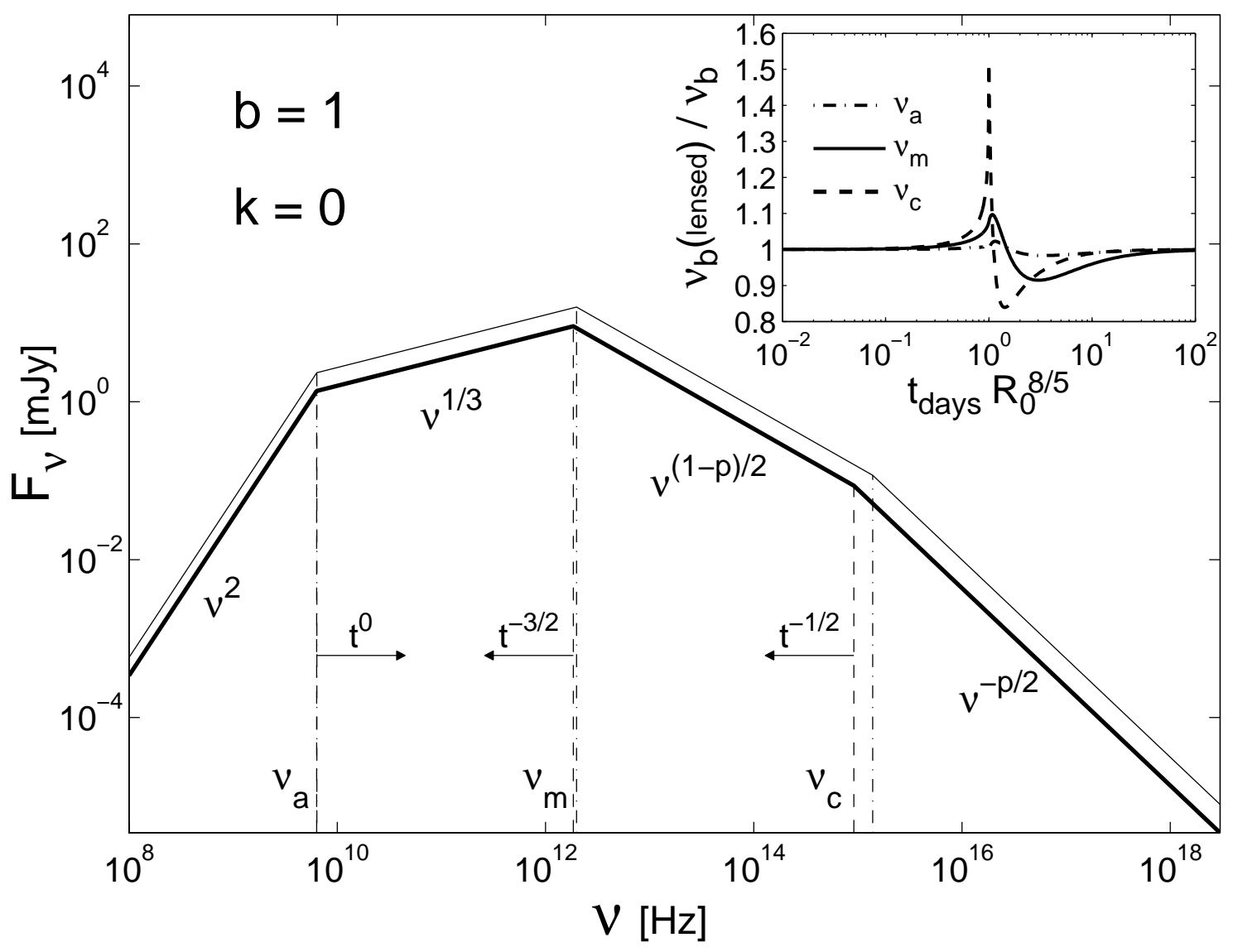

Fig. 1. - Typical broken power-law spectrum of a GRB afterglow at a redshift $z=1$ (boldface solid line). The observed flux density, $F_{\nu}$, as a function of frequency, $\nu$, is shown at an observed time $t_{\text {days }}=1$ for an explosion with a total energy output of $10^{52}$ erg in a uniform $\operatorname{ISM}(k=0)$ with a hydrogen density of $1 \mathrm{~cm}^{-3}$, and post-shock energy fractions in accelerated electrons and magnetic field of $\epsilon_{e}=0.1$ and $\epsilon_{B}=0.03$, respectively (using the scalings and notations of GS01). The thin solid line shows the same spectrum, when it is microlensed by an intervening star with $b=1$ and $R_{0}=1$ (see $\oint^{3}$ ). The insert shows the excess evolution of the break frequencies $\nu_{\mathrm{b}}=\nu_{a}, \nu_{m}$ and $\nu_{c}$ (normalized by their unlensed values) due to microlensing. 


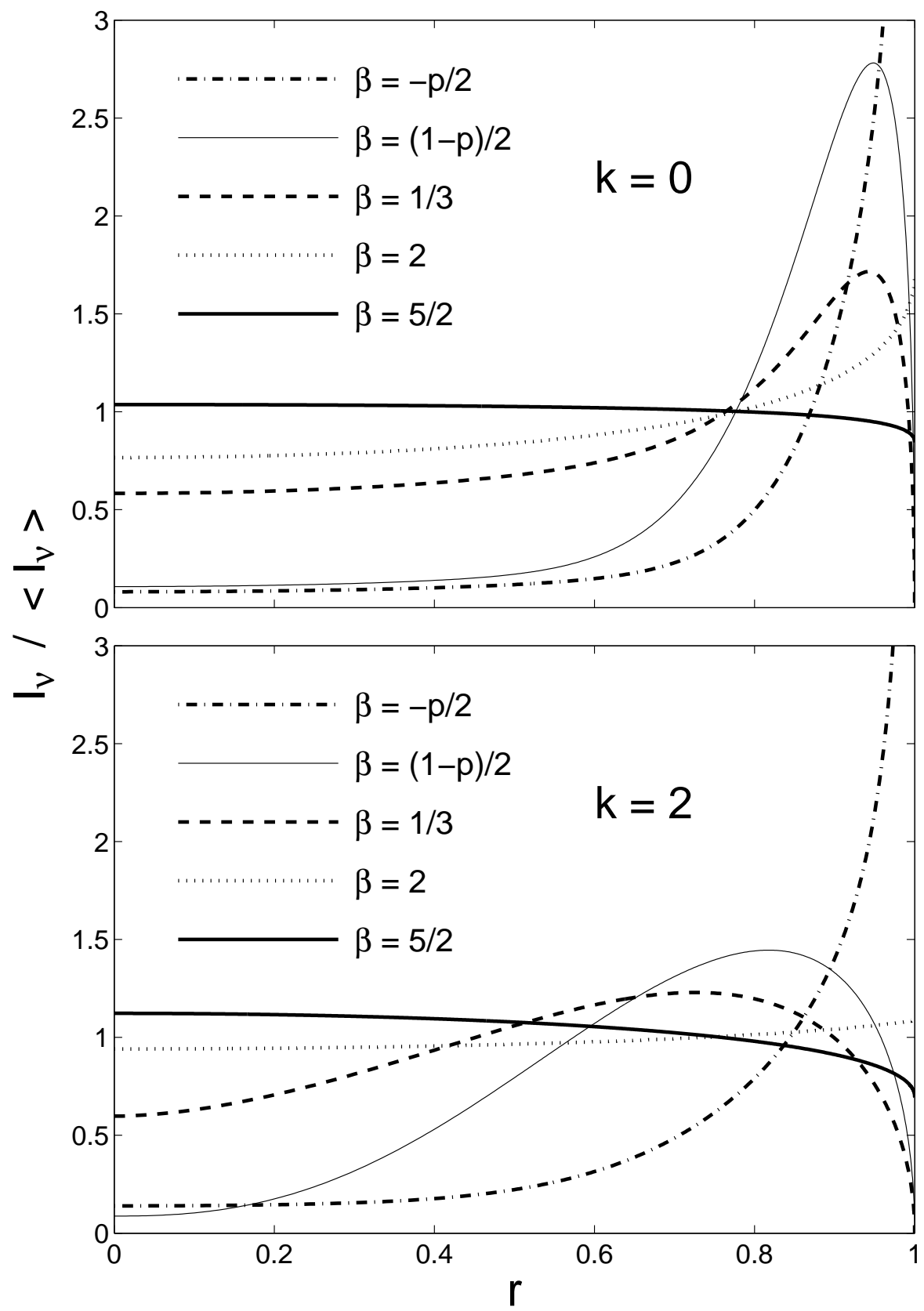

Fig. 2. - The surface brightness, normalized by its average value, as a function of the normalized radius, $r$, from the center of the image (where $r=0$ at the center and $r=1$ at the outer edge). The image profile changes considerably between different power-law segments of the afterglow spectrum, $F_{\nu} \propto \nu^{\beta}$. There is also a strong dependence on the density profile of the external medium, $\rho \propto R^{-k}$. 

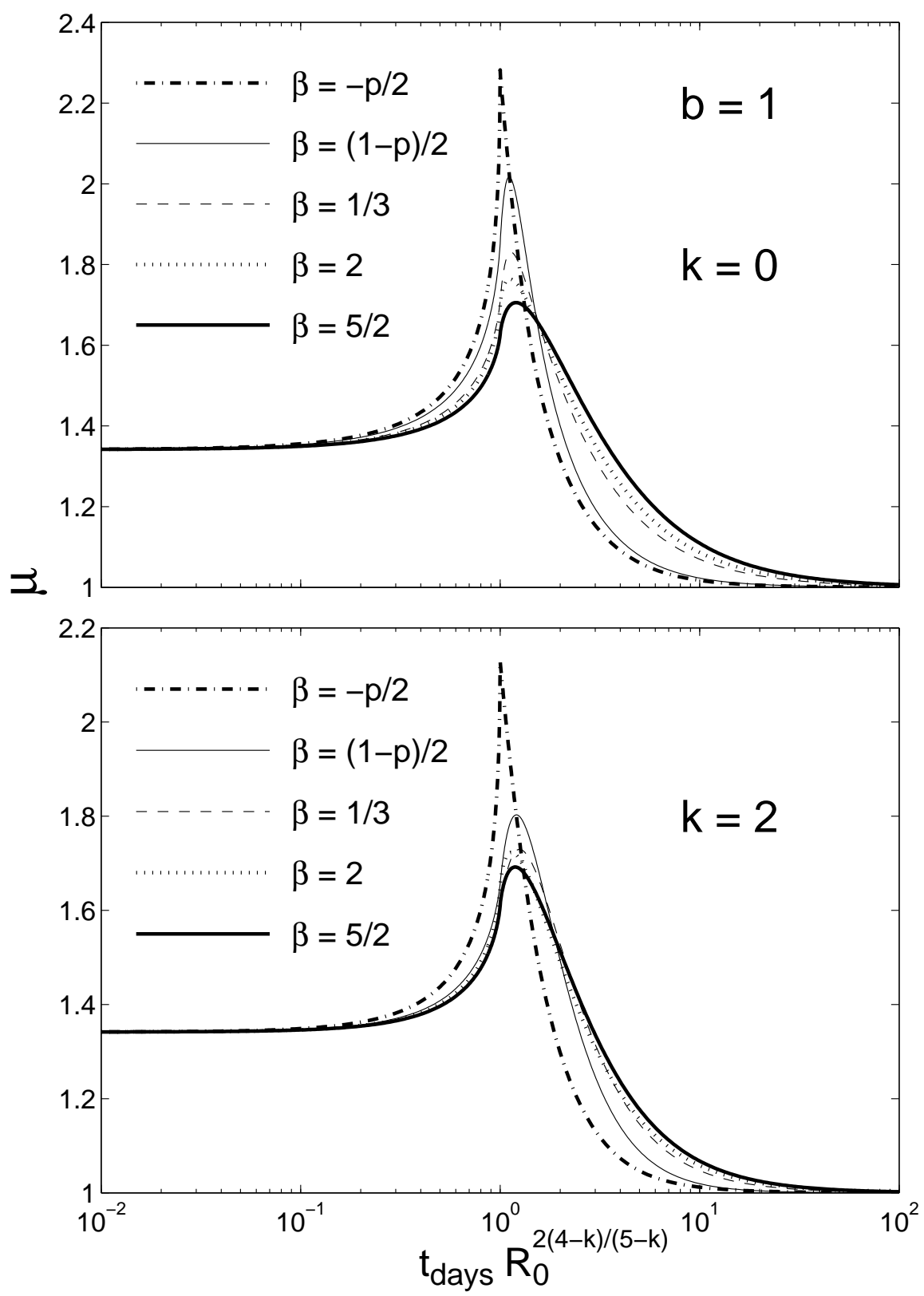

Fig. 3.- The magnification of the afterglow, $\mu$, as a function of normalized time, for different power-law segments, $F_{\nu} \propto \nu^{\beta}$, and different external density profiles $\left(\rho \propto R^{-k}\right.$ for $\left.k=0,2\right)$. The lens is assumed to be separated by one Einstein angle from the center of the source $(b=1)$. 